\title{
A novel iterative approach for mapping local singularities from geochemical data
}

\author{
Zhijun Chen ${ }^{1,2}$, Qiuming Cheng ${ }^{1,2,3}$, Jiaoguo Chen ${ }^{1,2}$, and Shuyun Xie ${ }^{1,4}$ \\ ${ }^{1}$ State Key Laboratory of Geological Processes and Mineral Resources, Wuhan 430074, Beijing 100083, China \\ ${ }^{2}$ Faculty of Earth Resources, China University of Geosciences, Wuhan Hubei 430074, China \\ ${ }^{3}$ Department of Earth and Space Science, Department of Geography, York University, Toronto, M3J 1P3, Canada \\ ${ }^{4}$ Faculty of Earth Sciences, China University of Geosciences, Wuhan Hubei 430074, China
}

Received: 14 February 2007 - Revised: 8 June 2007 - Accepted: 8 June 2007 - Published: 28 June 2007

\begin{abstract}
There are many phenomena in nature, such as earthquakes, landslides, floods, and large-scale mineralization that are characterized by singular functions exhibiting scale invariant properties. A local singularity analysis based on multifractal modeling was developed for detection of local anomalies for mineral exploration. An iterative approach is proposed in the current paper for improvement of parameter estimations involved in the local singularity analysis. The advantage of this new approach is demonstrated with de Wijs's zinc data from a sphalerite-quartz vein near Pulacayo in Bolivia. The semivariogram method was used to illustrate the differences between the raw data and the estimated data by the new algorithm. It has been shown that the outcome of the local singularity analysis consists of two components: singularity component characterized by local singularity index and the non-singular component by prefractal parameter.
\end{abstract}

\section{Introduction}

Singularities often carry significant information in signals and images. Detection and characterization of singularities form a crucial step in the analysis of various signals and image processing. Hence, the available time-frequency analysis methods, such as the short-time Fourier transform, the Wigner distribution, and the wavelet transform, are common detection tools (Yang et al., 2006). For example, Mallet and Hwang (1992) first introduced a method for detecting singularities in a time series signal by examining the evolution of the modulus maxima of the wavelet transform across the scales. The decay of the line of maxima can then be used to determine the regularity of the signal at a given time point. The measure of this decay is the Hölder exponent, also known as the Lipschitz exponent. Wavelet provides a power-

Correspondence to: Qiuming Cheng

(qiuming@yoku.ca) ful tool for extraction of information about the regularity of a signal. By examining the decay of the scalogram at specific points in time across all scales (frequencies), the regularity of the Hölder exponent can be determined. However, current research reveals that the singularities present in engineering signals are characterized not only by sudden modifications of amplitude and frequency, but also by sudden changes of the geometric shape of the signal. Here, we call the latter a singularity in the structure of the signal that has been often ignored. Yang et al. (2006) developed a method using the well-known singular value decomposition (SVD) method, information entropy, and principal component analysis techniques to identify singularities in signals, whether they were characterized by sudden changes in amplitude, structure, or frequency. Besides, when a signal is a function whose singularities are not isolated, measurements with finite numerical resolution are not sufficient to distinguish individual singularities. One attempt to compute singularity spectrum of multifractals is by taking advantage of their self-similarities (Mallet, 2003).

Since the pioneering work of Mandelbrot (1977, 1983), the concept of a fractal, a fast-moving research front coupled with concepts of complexity, criticality, and selforganization, has extended our usual ideas of classical geometry beyond those of points, lines, circles, and so on into the realm of irregular, disjoint, and singular structures $(\mathrm{Li}$, 2000). There are many phenomena in physics and other fields that are characterized by complicated singular measures or singular functions exhibiting self-similar scaling properties (Feder, 1988; Arneodo et al., 1995). More and more complex processes, such as multiplicative cascade processes, diffusion-limited aggregation, turbulence, and Brownian motion, have been suggested as having multifractal measures (Feder, 1988; Schertzer and Lovejoy, 1991; Evertsz and Mandelbrot, 1992). It has become a standard procedure to analyze fractals via the multifractal formalism, which was introduced to provide a statistical description of singular

Published by Copernicus Publications on behalf of the European Geosciences Union and the American Geophysical Union. 
measures in terms of thermodynamic functions such as the generalized fractal dimension $D_{q}$ and the singularity spectrum $f(\alpha) . D_{q}$ is intimately related to the spectrum of singularities $f(\alpha)$ by means of a Legendre transformation. This connection results from a deep analogy that links the multifractal and the thermodynamic formalism (Frisch and Parisi, 1985; Halsey et al., 1986). Therefore, $D_{q}$ and the spectrum of singularities $f(\alpha)$ are thermodynamic functions, i.e. statistical averages that provide only macroscopic information about the scaling properties of fractals. Recently, some localized approaches to the characterization of fractal objects have been proposed and developed. Arneodo et al. (1992, $1995,1998)$ provided a thermodynamics of fractals based on wavelet analysis, aimed towards a unified theory of singular distributions, including multifractal measures and multifractal functions. The wavelet transform modulus maxima (WTMM) method consists in building a partition function from the modulus maxima of the wavelet transform. By analyzing the behavior the wavelet transform of a fractal function versus the scale parameter along the WTMM lines, one can estimate the value of the local Hölder exponent. This method provides a natural generalization of the classical boxcounting techniques to fractal signals, the wavelets playing the role of "generalized boxes". Cheng (1999a) elaborated on a local singularity analysis based on multifractal modeling that provides a powerful tool for characterizing the local structural properties of spatial patterns. By analyzing the behavior of a singular measure versus the scale within small spatial-temporal intervals in the multifractal formalism, one can estimate the local Hölder exponent. This method provides a simple and direct strategy for detecting and characterizing singularities and has been successfully applied in many fields, such as anomaly enhancement and identification of geochemical data (Cheng, 2001a, b, 2004, 2006a, b, c), and texture analysis of remote-sensing images (Cheng, 1999a).

Cheng's local singularity analysis is briefly introduced in Sect. 2. In Sect. 3, we develop an iterative approach to improve the estimation of the local Hölder exponent. Section 4 is a case study. The usefulness of the new approach is demonstrated by application to the well-studied data on zinc concentration from a sphalerite-quartz vein near Pulacayo in Bolivia reported by de Wijs (1951), who also formulated the well-known de Wijsian model, which provides an early example of a multifractal (Mandelbrot, 1983; Agterberg, 2001). This zinc data set has been widely used in geostatistical and multifractal modeling (Matheron, 1962; Cheng and Agterberg, 1996; Cheng, 1994, 1997, 2000).

\section{Local singularities of multifractal measures}

The local singularity analysis based on multifractal modeling provided by Cheng (1999a) adopts the microcanonical method of determining the singularity spectrum directly from the data. More information about the principal of the method is referred to Cheng (2006c, 2007). We denote the mass by $\mu\left(B_{x}(\varepsilon)\right)$ with size $\varepsilon$ centered at $x$; the average density $\rho$ can then be expressed as $\rho\left(B_{x}(\varepsilon)\right)=\mu\left(B_{x}(\varepsilon)\right) / \varepsilon^{E}$, where $E$ is the Euclidean dimension ( $E=1$ for a one-dimensional problem and 2 for a two-dimensional problem). In general, however, fractal measures display multifractal properties in the sense that they scale differently from location to location. One is then led to consider a local scaling behavior following a power-law relationship, where

$\rho\left(B_{x}(\varepsilon)\right)=\mu\left(B_{x}(\varepsilon)\right) / \varepsilon^{E}=c(x) \varepsilon^{\alpha(x)-E}$

Here $\alpha(x)$ is the Hölder exponent, also termed the local singularity exponent in this paper; $\alpha(x)$ represents the singularity strength of the measure, while $c(x)$ determines the background magnitude. The smaller the local singularity exponent, the more singular the measure around $x$ and the "stronger" the singularity.

Keep in mind that the previous and further discussions are under an assumption that the local singularity convergence in average sense in a small area which is different from pointwise singularity. More discussions about the localization of singularity can be found in Cheng (2007). The local singularity exponent in Eq. (1) has the following properties (Cheng, 2001a, 2007):

(1) $\alpha(x)=E$ if and only if ("iff" for short) $\rho\left(B_{x}(\varepsilon)\right.$ ) is a constant, independent of the size $\varepsilon$; the measure is proportional to the area.

(2) $\alpha(x)<E$ iff $\rho\left(B_{x}(\varepsilon)\right)$ is a decreasing function of $\varepsilon$, $\rho\left(B_{x}(\varepsilon)\right)$ becomes very large when $\varepsilon$ becomes small, which implies a "convex" property and positive singularity of $\rho\left(B_{x}(\varepsilon)\right)$ in a small area centered around the given location.

(3) $\alpha(x)>E$ iff $\rho\left(B_{x}(\varepsilon)\right)$ is an increasing function of $\varepsilon$, $\rho\left(B_{x}(\varepsilon)\right)$ becomes very small when $\varepsilon$ becomes small, which implies a "concave" property and negative singularity of $\rho\left(B_{x}(\varepsilon)\right)$ in a small area centered around the given location.

Case (1) means nonsingular, and cases (2) and (3) correspond to a singular situation where $\rho\left(B_{x}(\varepsilon)\right)$ depends on the size $\varepsilon$. Therefore, the $\alpha$-value can be used to characterize the spatial structural properties of the density or measure function within small vicinity.

A window-based method for mapping local singularity can be used as follows (Cheng, 2006c). Given a location on a map, define a set of windows $A(r)$ (square, circular, rectangular, and elliptical) with variable window sizes $r_{\min }=r_{1}<r_{2}$ $\ldots<r_{i}<\ldots<r_{n}=r_{\max }(i=1,2, \ldots, n)$. Let $\varepsilon_{i}=r_{i} / r_{\max }$, since normalization is a geometrically invariant transformation and does not alter the measure. For each window size, calculate the mass value $\mu\left(A\left(\varepsilon_{i}\right)\right)$ or $\rho\left(B_{x}(\varepsilon)\right) \varepsilon^{E}$. The values $\mu\left(A\left(\varepsilon_{i}\right)\right)$ will show a linear trend with the linear size $\varepsilon_{i}$ on log-log paper, or $\log \left(\mu\left(A\left(\varepsilon_{i}\right)\right)\right)=c+\alpha \log \left(\left(\varepsilon_{i}\right)\right)$. Similar treatment with 
a sliding window at all locations on the map can create a singularity distribution map. The uncertainty related to the estimation of the singularity index can also be recorded and mapped. The use of different ranges of $\left[r_{\min }, r_{\max }\right]$ to ensure distinct power-law functions may yield singularities on different scales; for example, small-scale singularities may reflect local anomalies, whereas large-scale singularities may represent regional background variability.

\section{The iterative approach to local singularity analysis}

It is natural and simple to scan the support of $\mu$ by measuring $\alpha(x)$ at each location $x$ estimated by the slope of the curve of $\ln \left(\mu\left(B_{x}(\varepsilon)\right)\right)$ as a function of $\ln \varepsilon$. However, such a method would lead to some errors since, for any small area with linear size $\varepsilon, \mu\left(B_{x}(\varepsilon)\right)$ is the average values of many points which may have different singularity exponents. In Cheng's algorithm, it calculates the standard error and correlation coefficient involved in the estimation of singularity index. These statistics can be used to evaluate how good the estimation and only the results with statistical significance are used.

The difference between the $\alpha$-value and the object's Euclidean dimension is used to identify the local singularity in the above method. So, under conditions of finite numerical resolution, the key issue in the local singularity analysis is that how to improve the estimation of the $\alpha$-value whose bias may yield an incorrect interpretation. We notice that the model (1) involves the local coefficients ( $c$-values) accompanying the $\alpha$-values and c-value should be a nonsingular value (Cheng, 2005). However, if c-value is not completely nonsingular due to the calculation process, then the expected $\alpha$-value may be affected accordingly. This leads to the new idea of iterative approach. The goal is to improve the results so that the c-value does not contain singular component and therefore a-value reaches optimal. Therefore, we define $\alpha^{*}(x)$ and $c^{*}(x)$ as the optimal local singularity index and local coefficient, respectively, such that

$\rho(x)=c^{*}(x) \varepsilon^{\alpha^{*}(x)-\mathrm{E}}$

where $\rho(x)$ is the average density of a ball $B_{x}(\varepsilon)$ centered at $x$. We then have the following cases:

(1) $c^{*}(x)=\rho(x)$ as $\rho$ is nonsingular at $x$ with $\alpha^{*}(x)=E$.

(2) $c^{*}(x)<\rho(x)$ as $\rho$ is positive singular at $x$ with $\alpha^{*}(x)<E$.

(3) $c^{*}(x)>\rho(x)$ as $\rho$ is negative singular at $x$ with $\alpha^{*}(x)>E$.

So the $c$-values vary with location, and form a new data set whose fluctuation is smaller than that of the data set of $\rho$ values. The $c$-values can be taken as a coarse-scale approximation of the $\rho$-values, whose finest-scale information is characterized by the $\alpha$-values. $c$-value should be nonsingular component in theory, since $\alpha^{*}$ gives a characterization of the singularity (Cheng, 2005). The current paper takes the regularity of the $c$-values into account and provides a new approach to estimate $\alpha^{*}$ and $c^{*}$ by employing an iterative procedure. The model is expressed as

$c^{(k-1)}(x)=c^{(k)}(x) \varepsilon^{\alpha^{(k)}(x)-\mathrm{E}}$

where $k=0,1,2,3, \ldots$. Let $c^{(-1)}=\rho$ when $n=0$, and let $c^{(0)}$ be an ordinary $c$-value calculated in the first iterative equivalent to the results obtained by the ordinary non-iterative approach. The iteration function in Eq. (3) represents an iterative decomposition process producing a series $c^{(k)}$ and a series $\alpha^{(k)}$, and it also represents a reconstruction process which can be applied to the fractal interpolation problem.

A function should be constructed at every iteration $k$ to evaluate the closeness to the solution. Note that as $c^{(k)}$ becomes smoother after every iteration, $\alpha^{(k)}$ approaches $E$, and then $c^{(k-1)} / c^{(k)}=\varepsilon^{\alpha^{(k)}-E} \rightarrow 1$. Thus the correlation coefficient of the data set of successive local coefficients $R\left(c^{(k-1)}\right.$, $\left.c^{(k)}\right)$ can be calculated to control the precision of the solution. It is usual practice to specify a maximum number of iterations and a threshold $R_{0}$ for the local coefficient (e.g. 0.995 or bigger) as the stopping criteria for the procedure. At each iteration, an update is performed if $R\left(c^{(k-1)}, c^{(k)}\right)<R_{0}$. If this is not true when $k=n$, the iteration is stopped, and the raw data yield

$$
\begin{aligned}
\rho(x) & =c^{(0)}(x) \varepsilon^{\alpha(0)}(x)^{-\mathrm{E}} \\
& =c^{(1)}(x) \varepsilon^{\alpha(0)}(x)+\alpha^{(1)}(x)-\mathrm{E}-\mathrm{E} \\
& =c^{(n)}(x) \varepsilon^{\left[\alpha^{(0)}(x)+\sum_{k=1}^{n}\left(\alpha^{(k)}(x)-\mathrm{E}\right)\right]-\mathrm{E}}
\end{aligned}
$$

Hence, the optimal solution for $\alpha^{*}$ and $c^{*}$ is

$$
\left\{\begin{array}{c}
c^{*}(x)=c^{(n)}(x) \\
\alpha^{*}(x)=\alpha^{(0)}(x)+\sum_{k=1}^{n}\left(\alpha^{(k)}(x)-\mathrm{E}\right)
\end{array}\right.
$$

The iterative approach calculates not only the singularity exponents, but also coefficients which can be used for singular kriging interpolation and identification of anomalies (Cheng, 2000, 2005).

\section{Case study: de Wijs's zinc data}

The iterative approach to local singularity analysis has been tested on pedagogical examples, for example generalized devil's staircases and multiplicative cascade processes (Chen et al., 2005). This paper shows the results obtained on de Wijs' Zinc data from a mineral deposit. More examples including data generated by random multiplicative cascade processes will be given in the future papers. 

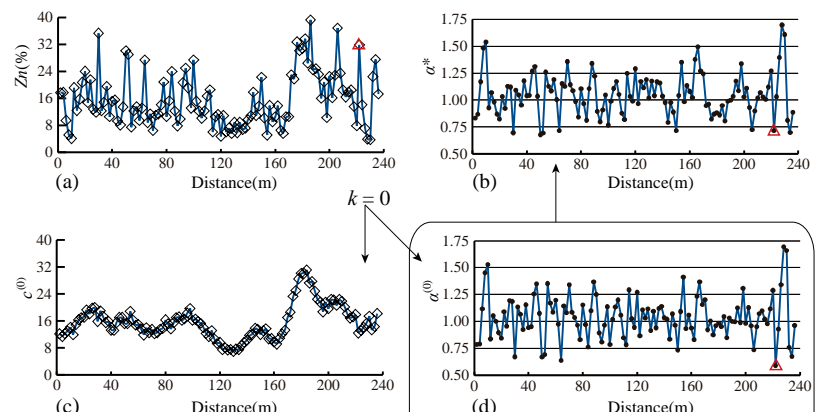

(d)
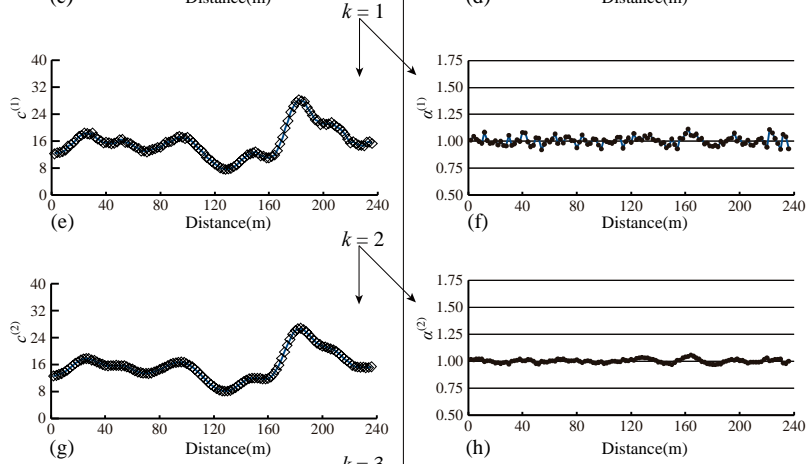

(h)
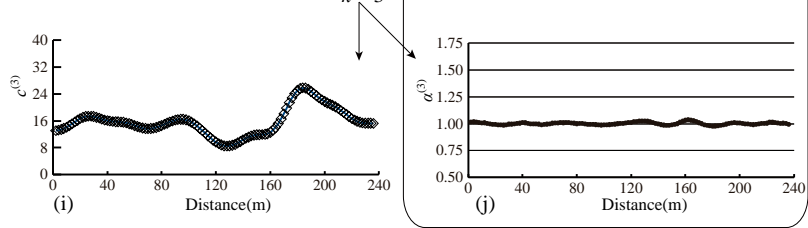

Fig. 1. Local singularity computation for the spatial series of 118 zinc percentage values sampled at $2 \mathrm{~m}$ intervals, from de Wijs. (a) Plot showing the raw data. (c) and (d) Plots showing the noniterative estimates of the local coefficient and local singularity index, respectively. (i) and (b) show the optimal estimates of the local coefficient and local singularity index, respectively, after three iterations. The downward arrows indicate the decomposition process and the iteration counter, the upward arrow starting from a diamond indicates the calculation of $\alpha^{*}$, and the red triangles indicate the location at a distance of $222 \mathrm{~m}$.

\subsection{Data set}

A classic multifractal data set is that of de Wijs (1951), who studied assay values from a sphalerite-quartz vein in the $\mathrm{Pu}$ lacayo Mine in Bolivia. These data were used for the purpose of validation of the new approach. Along a drift, 118 channel samples were cut across the vein at $2 \mathrm{~m}$ intervals. The massive vein filling averaged only $0.50 \mathrm{~m}$ in width but the wall rocks on both sides contained disseminated sphalerite, partly occurring in subparallel stringers. The channel samples were cut to a standard width of $1.30 \mathrm{~m}$, corresponding to the expected stopping width (Agterberg, 1974). These channel samples provided unbiased estimates of the zinc concentration in $2 \mathrm{~m}$-long blocks measured in the direction of the vein. De Wijs showed that the 118 zinc values were approximately log-normally distributed, whereas Cheng and Agter-
Table 1. Summary statistics for the de Wijs zinc data set, and results obtained by local singularity analysis.

\begin{tabular}{lrrrrr}
\hline & $\ln (\mathrm{Zn})$ & $\ln \left(c^{(0)}\right)$ & $\alpha^{(0)}$ & $\ln \left(c^{*}\right)$ & $\alpha^{*}$ \\
\hline Max & 3.6712 & 3.4372 & 1.6931 & 3.2501 & 1.6973 \\
Min & 1.3083 & 1.9383 & 0.5909 & 2.1399 & 0.6775 \\
Mean & 2.6124 & 2.7026 & 1.0385 & 2.7198 & 1.0475 \\
Trimmed mean & 2.632 & 2.7088 & 1.0298 & 2.7229 & 1.0368 \\
Std. deviation & 0.5359 & 0.3198 & 0.2014 & 0.2607 & 0.1989 \\
Skewness & -0.2438 & -0.1286 & 0.4974 & -0.1204 & 0.5616 \\
\hline
\end{tabular}

berg (1996) showed that these data followed a multifractal distribution. Local singularity analysis and multifractal interpolation were first applied to this data set by Cheng (2000).

\subsection{Calculation of singularities}

Figure 1a shows the spatial series of 118 zinc percentage values sampled at $2 \mathrm{~m}$ intervals from de Wijs. The iterative approach to local singularity analysis was applied to achieve optimization of the patterns in this data. We set five interval windows, whose half length ranged from $r_{\min }=1 \mathrm{~m}$ through 3,5 , and $7 \mathrm{~m}$ to $r_{\max }=9 \mathrm{~m}$, and the stop loop condition was $R_{0}=0.995$. For each window, the average value of the zinc percentage concentration was calculated by averaging the values of all samples in the interval. We then generated two sets of values: the average concentration values $\rho_{i}$ (or length-metal $\mu_{i}=\rho_{i} \varepsilon_{i}$ ) and the normalized sizes of the windows $\varepsilon_{i}=r_{i} / r_{\max }(i=1,2, \ldots, 5)$. The estimation of the $\alpha$-value and $c$-value was performed by a least-squares linear regression, which gave these values as the slope and $y$-intercept, respectively, of the scaling curves of $\ln \left(\mu_{i}\right)$ vs. $\ln \left(\varepsilon_{i}\right)$. In addition, the coefficients $R^{2}$ related to the linear fitting were also calculated.

\subsection{Results and discussion}

Figure 1 shows the decomposition and reconstruction processes of the local singularity analysis using the iterative approach. The optimal number of iterations was 3 , and the last successive correlation coefficient $R\left(c^{(2)}, c^{(3)}\right)$ reaches 0.997 (Fig. 2). The range of the singularity values $\Delta \alpha \neq 0$ (Fig. 1d) in the initial (noniterative) decomposition process indicates that de Wijs's zinc data (Fig. 1a) show the multifractality, and the range of the singularity values $\Delta \alpha \rightarrow 0$ (Fig. 1j) in the third decomposition process indicates that the data in Fig. 1g can be regarded as a nonfractal. It is obvious that the $c$-value curve becomes increasingly smooth, and the $\alpha$-values approach the Euclidean dimension $E=1$. Figures $1 \mathrm{c}$ and $\mathrm{d}$ show the noniterative estimates of the local coefficient and the local singularity exponent, respectively, and Figs. $1 \mathrm{i}$ and $\mathrm{b}$ show the optimal estimates of these quantities after three iterations. The frequency distributions of majority values of $\alpha^{*}$ and $\alpha^{(0)}$ both approximately follow normal 


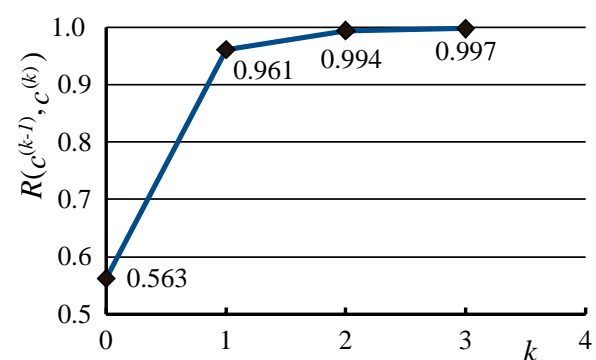

Fig. 2. Correlation coefficients of successive $c$-values, trending to 1 with increasing number of iterations.

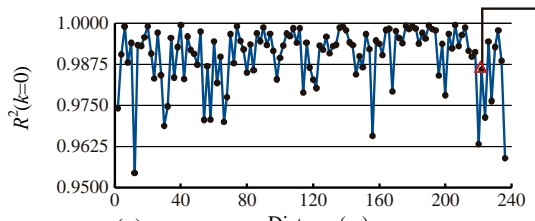

(a)
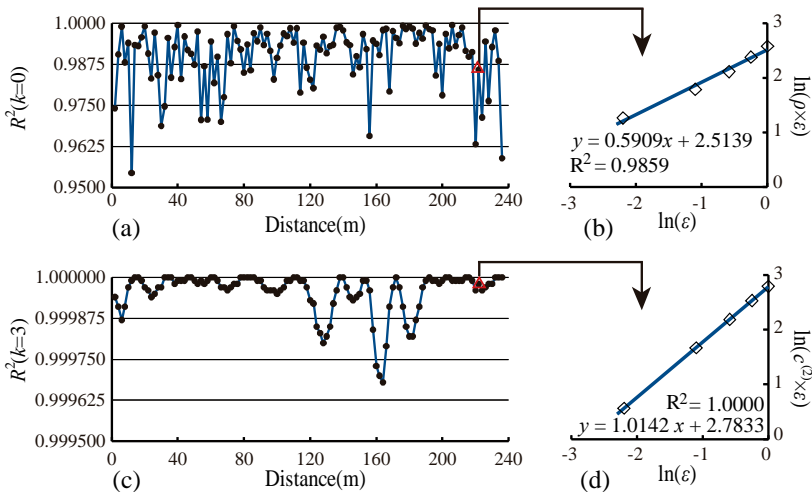

Fig. 3. Significance validation for the least-squares linear regression on a log-log plot of $\ln (\mu)$ vs. $\ln (\varepsilon)$. (a) and (c) show the coefficients $R^{2}$ for the noniterative decomposition and the third decomposition, respectively. (b) and (d) show the $\log -\log$ plot and the linear fit for the location at $222 \mathrm{~m}$ in the de Wijs data; (b) is for the noniterative decomposition and (d) is for the third decomposition.

distributions with nearly the same mean. However, the standard deviation of $\alpha^{*}$ is appreciably smaller than that of $\alpha^{(0)}$, and the standard deviation of $\ln (\mathrm{Zn}), \ln \left(c^{(0)}\right)$, and $\ln \left(c^{*}\right)$ also decrease (Table 1), which implies that the iterative approach has a tendency to make a conservative estimate of $\alpha^{*}$ and $c^{*}$. $c^{*}$ has smaller extreme-high and larger extreme-low values as compared with $c^{(0)}$, which means that the iterative approach has more power to remove the effects of extreme values or outliers in the raw data. The significance validation for the least-squares linear regression is shown in Fig. 3. We find that the coefficients $R^{2}$ tend to 1 , which means that the fitting is significant; in addition, the fluctuation of the coefficients $R^{2}$ becomes smaller and smaller as the number of iterations increases.

To reveal the spatial properties of the $c$-values, we used semivariogram analysis, which has been used widely to quantify the spatial association and spatial variability of spatial phenomena (Gringarten and Deutsch, 2001). The issue of modeling the semivariogram of de Wijs's zinc data has been discussed by many researchers (Matheron, 1962; Agterberg, 1974; Cheng, 1994; Cheng and Agterberg, 1996). Matheron

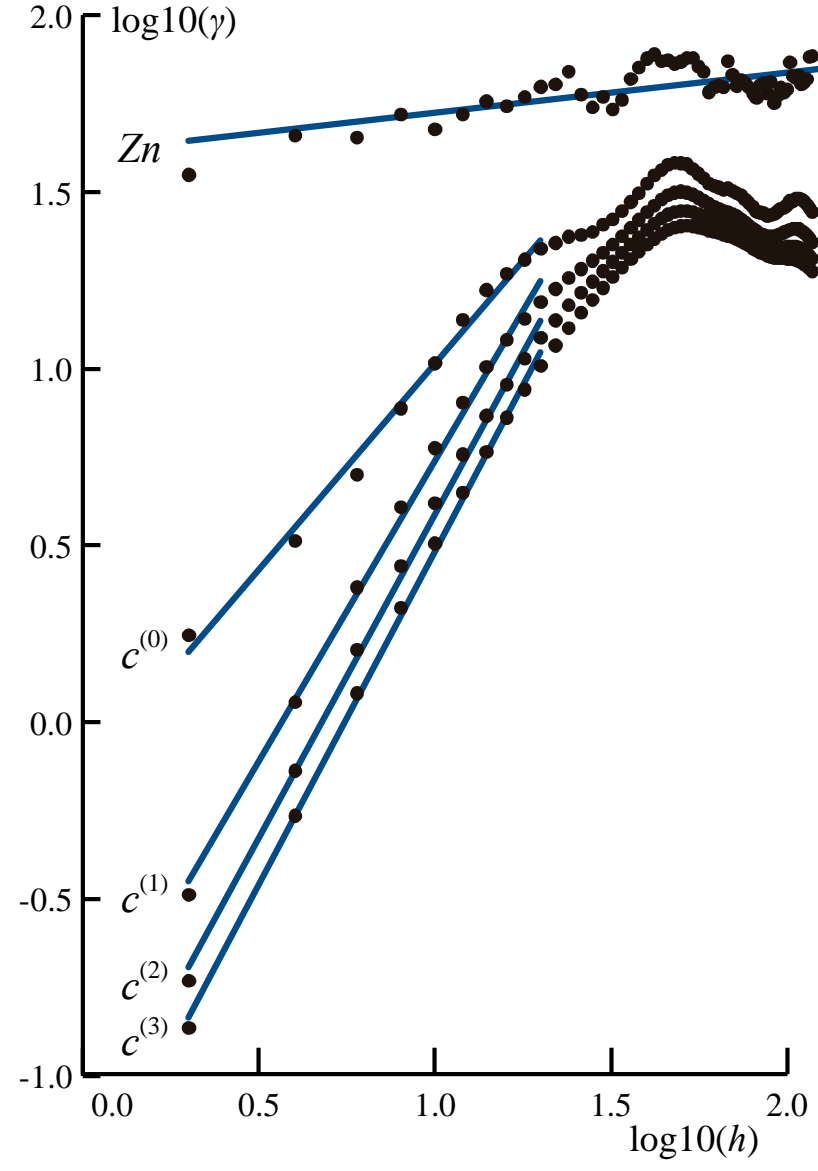

Fig. 4. Log-log (base 10) plot of the empirical semivariograms against lag, and modeled linear fits for zinc and $c$-value data. Fit for $\mathrm{Zn}: \beta=0.11, R^{2}=0.6959$. Fit for $c^{(0)}: \beta=1.17, R^{2}=0.9959$. Fit for $c^{(1)}: \beta=1.70, R^{2}=0.9980$. Fit for $\mathrm{c}^{(2)}: \beta=1.83, R^{2}=0.9986$. Fit for $\mathrm{c}^{(3)}: \beta=1.88, R^{2}=0.9993$.

(1962) operated on logarithmically transformed data and provided the well-known logarithmic model. Recent studies have indicated that the de Wijs data follow a multifractal distribution and that they can be modeled by a theoretical multifractal semivariogram, derived by Cheng and Agterberg (1996) with $\tau(2)=0.979 \pm 0.019$ :

$$
\begin{gathered}
\gamma(h)=30.73+360.76\left\{1-\frac{1}{2}\left[\left(\frac{h}{2}+1\right)^{1.979}\right.\right. \\
\left.\left.-2\left(\frac{h}{2}\right)^{1.979}+\left(\frac{h}{2}-1\right)^{1.979}\right]\right\}
\end{gathered}
$$

Empirical semivariograms of the raw data and $c^{(k)}$ data (where $k=0,1,2,3$ ) were calculated with lags of $2 \mathrm{~m}, 4 \mathrm{~m}$, $6 \mathrm{~m}, \ldots, 118 \mathrm{~m}$. The empirical semivariograms of the $\mathrm{Zn}$ data fluctuate periodically while ascending without an obvious sill, so that the deviations between the experimental semivariogram and the theoretical curve are relatively large. 

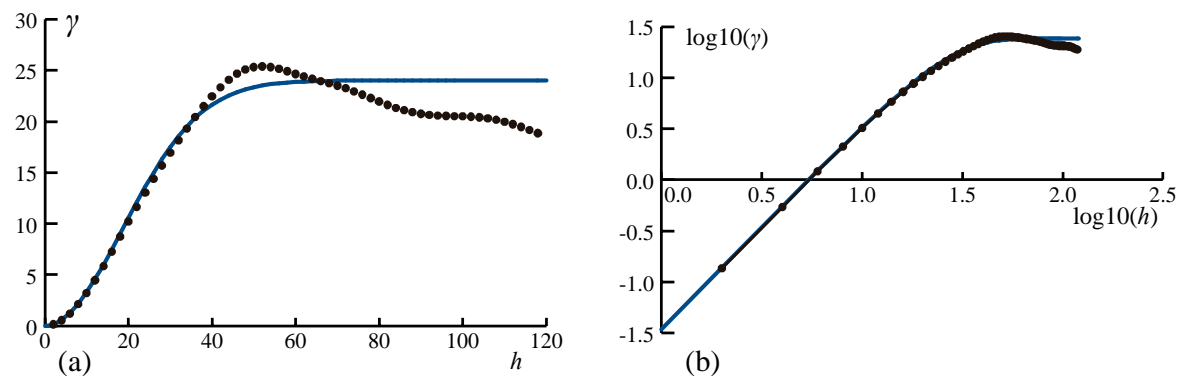

(b)

Fig. 5. Best fit of Gaussian model weighted by $N(h)[\gamma(h ; \theta)]^{-2}$ to the empirical semivariogram of the $c^{(3)}$ data. $\gamma(h)=24.00\left(1-\exp \left(-\frac{h^{2}}{26.24^{2}}\right)\right)$. (a) linear mode; (b) log-log mode (base 10). The model is Gaussian with zero nugget effect and a practical range of $46.14 \mathrm{~m}$. The sum of the weighted square differences is 0.0108 and the Akaike information criterion is -532.6860 .

The $\mathrm{Zn}$ data have an obvious nugget effect, which accounts for large portions of the approximate "sill"; this means that the regionalized variable is generally not continuous and that microstructure with a very short range could not be revealed with the current sampling support. Thus the behavior at the origin is maybe very complex; probably the process is highly random, even nonlinear and chaotic, leading to variations without any spatial correlation structure. However, the experimental semivariogram of the $c$-values can be fitted by a transitive semivariogram model very well, with a small nugget effect.

By definition, a semivariogram is a measure of the average dissimilarity or roughness between observations as a function of the separation vector $\boldsymbol{h}$ (Goovaerts, 1999; Herafeld and Overbeck, 1999). Assume the existence of an autocorrelation function $C(h)$ of a profile (a graph $f:[a, b]$ $\rightarrow R) h=|\boldsymbol{h}|$ for a lag distance between two points without considering the direction. There is then a constant $c>0$ and $1 \leq s \leq 2$, such that

$\gamma(h)=C(0)-C(h) \approx c h^{4-2 s}=c h^{\beta}$

The fractal box dimension $D$ estimated by the variogram method is then equal to $s$ (Falconer, 1990). Therefore, when $\gamma(h)$ is plotted against $h$ on log-log paper, the fractal box dimension $D$ is equal to $2-\beta / 2$. The fractal dimension can reveal the spatial complexity of a spatial pattern. Commonly, the more complicated the spatial pattern is, the higher is the fractal dimension. The Hurst exponent $H$ is an another important index for characterizing the scale-invariant correlation, which often used in rescaled range $(\mathrm{R} / \mathrm{S})$ analysis. $H$ is related to the fractal dimension $D$ as follows: $D=2-H$ for profiles, $D=3-H$ for surfaces, $D=4-H$ for volumes, etc. (Carr, 1997). For the 1-D case, $H=0.5$ is consistent with the Brown noise model; $H=0(D=2)$ corresponds to pure white (random) noise; and $H=1(D=1)$ corresponds to nonfractal, ideal geometric behavior. The smaller the value of $H$ is, the more random are the data. So we can analyze the properties of $D$ and $H$ for the raw $\mathrm{Zn}$ data and for $c^{(0)}, c^{(1)}, c^{(2)}$, and $c^{(3)}$.
The empirical semivariogram of the $\mathrm{Zn}$ data was plotted on log-log paper and fitted by a straight line segment with slope $\beta=0.11$. The Hurst dimension and fractal box counting dimension of the $\mathrm{Zn}$ data were 0.055 and 1.945 , respectively (Fig. 4). However, the dots for the $c^{(0)}, c^{(1)}, c^{(2)}$, and $c^{(3)}$ data could be divided approximately into two groups, which means that the power law in Eq. (7) is valid within a finite range. So only the dots near the origin (10 dots within $20 \mathrm{~m}$ ) were selected and fitted by line segments (Fig. 4). The result of the fit shows that the linear behavior near the origin is significant for all of the $c^{(0)}, c^{(1)}, c^{(2)}$, and $c^{(3)}$ data and that all slopes of the $c$-value data are bigger than that of the $\mathrm{Zn}$ data. What is more, the slopes tend to 2 with increasing iteration number. The Hurst dimensions of the $c^{(0)}$ and $c^{(3)}$ data are 0.585 and 0.940 , respectively, and the fractal box counting dimensions are 1.415 and 1.060 , respectively. This brings out the fact that the $c^{(0)}$ data still suffer from some singularity, since they has a larger fractal dimension and smaller Hurst dimension, and it is reasonable that $c^{(3)}$ should be considered as $c^{*}$ with a regularity property. The empirical semivariograms of the $c^{(3)}$ data can be best fitted by a Gaussian model without a nugget effect (Fig. 5),

$\gamma(h)=24.00\left(1-\exp \left(-\frac{h^{2}}{26.24^{2}}\right)\right)$

A Gaussian model has a parabolic form near the origin. In theory, this model is associated with an infinitely differentiable stationary random function (SRF) and thus is extremely regular (Chilès and Delfiner, 1999). The regularity gives the SRF a deterministic character, in that knowledge of the value of the SRF at 0 and the values of its partial derivatives of all orders determines the value of the SRF at any location $\boldsymbol{x}$ (Chilès and Delfiner, 1999). Such regularity is hardly ever encountered in the earth sciences; however, this type of data will probably be available when the raw data are processed by the iterative approach that we have provided.

The coefficient $c^{*}$ is so regular that the interpolation problem for it is easy to solve by using kriging or other interpolation methods such as inverse distance weighted (IDW) 
interpolation. Suppose that $\hat{c}^{*}$ is a result of interpolation of $c^{*}$, Therefore the raw data can be interpolated by

$\hat{\rho}(x)=\hat{c}^{*} \varepsilon^{\hat{\alpha} *-\mathrm{E}}$

where $\hat{\alpha}^{*}$ is the local singularity exponent corresponding to $\hat{c}^{*}$. The interpolation function in Eq. (9) takes the scale and the local singularity into consideration, whereas the conventional interpolation methods have a smoothing effect. A singular kriging interpolation technique based on geostatistics and local singularity analysis has been proposed and discussed by Cheng (1999b, 2000, 2005).

\section{Conclusions}

There are many good characteristics of the local singularity exponent, and so in general it can be used in many fields, but it is especially useful in the detection of singularities in signals, identification of anomalies in geochemical data, and the singular interpolation technique. Local singularity analysis based on multifractal modeling exhibits more convenience and feasibility in practical applications in geochemistry than do the available time-frequency analysis tools, such as wavelet transforms. It is essential to note that the key to the application of singularity analysis is the improvement of the estimation of the local singularity exponents. The local value of the coefficient $c$, as well as the $\alpha$-value, plays a central role in local singularity analysis.

The iterative approach to local singularity analysis proposed in this paper focuses on investigation of the regularity of $c$-values to improve the estimation of $\alpha$-values. It has been demonstrated by the case study of the de Wijs's zinc data that this approach is superior to the noniterative approach. The latter can be considered as a special case of the former. We have explored the differences between the raw data and the local coefficients in terms of the spatial association and regularity properties using semivariogram analysis. Ideally, the $c^{*}$ obtained by the iterative approach is nonfractal, without a nugget effect and with $D=1$ and $H=2$, whereas the raw data will have a bigger nugget effect, a bigger $D$-value, and a smaller $H$-value if it follows a multifractal distribution. The $c^{*}$ data are so regular that they can be best fitted by a Gaussian model. It is not unreasonable to expect that combining spatial variability with singularity analysis will improve interpolation results significantly, especially for observed data with singularities.

The iterative approach has the shortcomings that it is timeconsuming and that the spread of edge effects is unavoidable with the iterations. Also, the current procedure for local singularity analysis does not consider the shape of the windows used; monotonic windows are arbitrarily slid to any location to calculate the $\alpha$-value and $c$-value in the above procedure. However, it is a crucial issue to specify suitable windows (in terms of size and shape) with different locations, since the object of study is usually anisotropic and inhomogeneous.
Further study will be devoted to a practical algorithm for the estimation of local singularity exponents which will take edge effects, local anisotropic scaling invariance at arbitrary locations, etc. into consideration.

Acknowledgements. The authors thank F. Agterberg at the Geological Survey of Canada and an anonymous reviewer for their critical reading of this manuscript with constructive comments. This research was jointly supported by Student Grant awarded by the IAMG (International Association of Mathematical Geology), a Distinguished Young Researcher Grant (40525009) and a Strategic Research Grant (40638041) awarded by the Natural Foundation of Science of China and a 863 High Tech program (2006AA06Z115) by the Ministry of Science and Technology of China.

Edited by: A. Tarquis

Reviewed by: F. Agterberg and another anonymous referee

\section{References}

Agterberg, F. P.: Geomathematics: mathematical background and Geo-science Application, Elsevier scientific publishing company, Amsterdam, 317-324, 1974.

Agterberg, F. P.: Multifractal Simulation of Geochemical Map Patterns, Journal of China University of Geosciences, 12, 31-39, 2001.

Arneodo, A., Argoul, F., Bacry, E. Elezgaray, J., Freysz, E., Grasseau, G., Muzy, J. F., and Pouligny, B.: Wavelet transform of fractals, in: Wavelets and applications: Proceedings of the International Conference, edited by: Meyer, Y., Marseille, France, May 1989, Springer-Verlag, Paris, 287-352, 1992.

Arneodo, A., Bacry, E., and Muzy, J. F.: The thermodynamics of fractals revisited with wavelets, Physica A, 213, 232-275, 1995.

Arneodo, A., Audit, B., Bacry, E., Manneville, S., Muzy, J. F., and Roux, S. G.: Thermodynamics of fractal signals based on wavelet analysis: application to fully developed turbulence data and DNA sequences, Physica A, 254, 24-45, 1998.

Carr, J. R.: Statistical self-affinity, fractal dimension, and geologic interpretation, Eng. Geology, 48, 269-282, 1997.

Chen, Z. J., Cheng, Q., and Chen, J. G.: Significance of Fractal Measure in Local Singularity Analysis of Multifractal Model, in: Proceedings of IAMG'05: GIS and Spatial Analysis, edited by: Cheng, Q. and Bonham-Cater, G., International Association for Mathematical Geology, China University of Geosciences Printing House, Wuhan, vol. 1, 475-480, 2005.

Cheng, Q. and Agterberg, F. P.: Multifractal modeling and spatial statistics, Math. Geology, 28, 1-16, 1996.

Cheng, Q.: Multifractal modeling and spatial analysis with GIS: gold potential estimation in the Mitchell-Sulphurets area, Northwestern British Columbia, Ph.D. thesis, University of Ottawa, Ottawa, 268 pp, 1994.

Cheng, Q.: Multifractal modeling and lacunarity analysis, Math. Geology, 29, 919-932, 1997.

Cheng, Q.: Multifractality and spatial statistics, Comput Geosci., 25, 949-961, 1999a.

Cheng, Q.: Multifractal interpolation, in: Proceedings of the Firth Annual Conference of the International Association for Mathematical Geology, edited by: Lippard, S. J., Naess, A., and Sinding-Larsen, R., Trondheim, Norway, vol. 1, 245-250, 1999b. 
Cheng, Q.: Interpolation by means of multiftractal, kriging and moving average techniques, in: CD Proceedings of GAC/MAC meeting GeoCanada2000, 29 May to 2 June, Calgary, http: //www.gisworld.org/gac-gis/geo2000.htm, 2000.

Cheng, Q.: Singularity analysis for image processing and anomaly enhancement, in: Proceedings IAMG'01, International Association for Mathematical Geology, Cancun, Mexcico, 6-12 September, CD-ROM, 2001a.

Cheng, Q.: Multifractal and geostatistical methods for exploration geochemical anomaly texture and singularity analysis, Earth Science - Journal of China University of Geosciences (in Chinese with English abstract), 26, 161-166, 2001b.

Cheng, Q.: Quantifying the generalized self-similarity of spatial patterns for mineral resources assessment, Earth Science - Journal of China University of Geosciences (in Chinese with English abstract), 29, 733-744, 2004.

Cheng, Q.: A new model for incorporating spatial association and singularity in interpolation of exploratory data, in: Geostatistics Banff 2004, Quantitative Geology and Geostatistics, Springer Press, edited by: Leuangthong, O. and Deutsch, C. V., Netherlands, 14, 1017-1025, 2005.

Cheng, Q.: GIS based fractal/multifractal anomaly analysis for modeling and prediction of mineralization and mineral deposits, in: GIS Application in the Earth Sciences - GAC Special Paper, Geological Association of Canada Special Book, edited by: Harris, J. R., 285-296, 2006 a.

Cheng, Q.: Singularity - generalized self-similarity - fractal specturm (3S) Model, Earth Science - Journal of China University of Geosciences (in Chinese with English abstract), 31, 337-348, 2006b.

Cheng, Q.: Mapping singularities with stream sediment geochemical data for prediction of undiscovered mineral deposits in Gejiu, Yunnan Province, China, Ore Geology Reviews, in press, doi:10.1016/j.oregeorev.2006.10.002, 2006c.

Cheng, Q.: Multifractal imaging filtering and decomposition methods in Space, Fourier Frequency, and Eigen Domains, Nonlin. Processes Geophys., 14, 293-303, 2007, http://www.nonlin-processes-geophys.net/14/293/2007/.

Chilès, J. P. and Delfiner, P.: Geostatistics: Modeling Spatial Uncertainty, John Wiley \& Sons, Inc., New York, 694 pp., 1999.

de Wijs, H. J.: Statistics of ore distribution, part I: Geologie en Mijnbouw, 30, 365-375, 1951.
Everstz, C. J. G. and Mandelbrot, B. B.: Multifractal measures, in: Chaos and Fractals, edited by: Peitgen, H., Jürgens, H., and Saupe, D., Springer, Berlin, 921-953, 1992.

Falconer, K.: Fractal Geometry: Mathematical Foundations and Applications, John Wiley \& Sons Ltd., Chichester, 288 pp., 1990.

Feder, J.: Fractals, Plenum, New York, 283 pp., 1988.

Frisch, U. and Parisi, G.: Fully developed turbulence and intermittency, in: Turbulence and Predictability of Geophysical Flows and Climate Dynamics, edited by: Ghil, M., Benzi, R., and Parisi, G., Amsterdam, North Holland, 84-88, 1985.

Goovaerts, P.: Geostatistics in soil science: state-of-the-art and perspectives, Geoderma, 89, 1-45, 1999.

Gringarten, E. and Deutsch, C. V.: Teacher's Aide: Variogram Interpretation and Modeling, 33, 507-534, 2001.

Halsey, T. C., Jensen, M. H., Kadanoff, L. P., Procaccia I., and Shraimann, B.: Fractal measures and their singularities: the characterization of strange sets, Phys. Rev., A, 33, 1141-1151, 1986.

Herzfeld, U. C. and Overbeck, C.: Analysis and simulation of scaledependent fractal surfaces with application to seafloor morphology, 25, 979-1007, 1999.

Li, B. L.: Fractal geometry applications in description and analysis of patch patterns and patch dynamics, Ecol. Model., 132, 33-50, 2000.

Mallet, S. and Hwang, W. L.: Singularity detection and processing with wavelets, IEEE Trans. information Theory, 38, 617-643, 1992.

Mallet, S.: A wavelet Tour of Signal Processing (2nd), China Machine Press, Beijing, 637 pp, 2003.

Mandelbrot, B. B.: Fractals: Form, Chance, and Dimension, W H Freeman \& Co. New York, 1977.

Mandelbrot, B. B.: The Fractal Geometry of Nature, W H Freeman \& Co., New York, 468 pp., 1983.

Matheron, G.: Traite de Geostatistique Appliquee, Tome 1, Memoires du Bureau de Recherches Geologiques. et Minieres, no. 14, Editions Technip, Paris, 334 pp, 1962.

Schertzer, D. and Lovejoy, S.: Non-linear variability in geophysics, Kluwer Academic Publ., Dordrecht, 318 pp., 1991.

Yang, W. X., Hull, J. B., and Seymour, M. D.: Detecting the singularities in engineering signals, J. Mat. Processing Technol., 175, 439-445, 2006. 